\title{
The Co-Occurrence of Chronic Diseases and Geriatric Syndromes: The Health and Retirement Study
}

\author{
Pearl G. Lee, MD,$^{* \neq}$ Christine Cigolle, MD, MPH, ${ }^{\dagger \neq}$ and Caroline Blaum, MD, MS ${ }^{* \ddagger}$
}

OBJECTIVES: To analyze the co-occurrence, in adults aged 65 and older, of five conditions that are highly prevalent, lead to substantial morbidity, and have evidencebased guidelines for management and well-developed measures of medical care quality.

DESIGN: Secondary data analysis of the 2004 wave of the Health and Retirement Study (HRS).

SETTING: Nationally representative health interview survey.

PARTICIPANTS: Respondents in the 2004 wave of the HRS aged 65 and older.

MEASUREMENTS: Self-reported presence of five index conditions (three chronic diseases (coronary artery disease, congestive heart failure, and diabetes mellitus) and two geriatric syndromes (urinary incontinence and injurious falls)) and demographic information (age, sex, race, living situation, net worth, and education).

RESULTS: Eleven thousand one hundred thirteen adults, representing 37.1 million Americans aged 65 and older, were interviewed. Forty-five percent were aged 76 and older, $58 \%$ were female, $8 \%$ were African American, and $4 \%$ resided in a nursing home. Respondents with more conditions were older and more likely to be female, single, and residing in a nursing home (all $P<.001$ ). Fifty-six percent had at least one of the five index conditions, and $23 \%$ had two or more. Of respondents with one condition, $20 \%$ to $55 \%$ (depending on the index condition) had two or more additional conditions.

CONCLUSION: Five common conditions ( 3 chronic diseases, 2 geriatric syndromes) often co-occur in older adults, suggesting that coordinated management of comorbid conditions, both diseases and geriatric syndromes, is important. Care guidelines and quality indicators, rather than considering one condition at a time, should be developed to address comprehensive and coordinated management of

From the * Departments of Internal Medicine; ${ }^{\dagger}$ Family Medicine, University of Michigan, Ann Arbor, Michigan; and ${ }^{\ddagger}$ Veterans Affairs Ann Arbor

Healthcare System Geriatric Research, Education and Clinical Center, Ann Arbor, Michigan.

Address correspondence to Pearl G. Lee, Department of Internal Medicine, University of Michigan 300 N. Ingalls, Room 920, Ann Arbor, MI 48109-

2007. E-mail: pearllee@umich.edu

DOI: 10.1111/j.1532-5415.2008.02150.x co-occurring diseases and geriatric syndromes. J Am Geriatr Soc 57:511-516, 2009.

Key words: disease; incontinence; fall

$\mathrm{O}$ Ider adults with multimorbidity, or co-occurrence of multiple chronic diseases, have poorer self-rated health and functional status, greater utilization of health care, and potentially earlier mortality than older adults with single diseases. ${ }^{1}$ Multimorbidity is common in the older adult population. It has been reported that $65 \%$ of Medicare beneficiaries have two or more chronic diseases and $43 \%$ have three or more; ${ }^{2}$ other studies have found that more than $50 \%$ of older adults have multimorbidity. ${ }^{3,4}$ Furthermore, having newly reported chronic diseases has been associated with risk of functional dependency. ${ }^{5}$ The research reported in this article adds to the study of multimorbidity by examining the co-occurrence of chronic diseases with geriatric syndromes.

Geriatric syndromes, such as falls and urinary incontinence (UI), are conditions that have not traditionally been considered to be chronic diseases; nonetheless, geriatric syndromes have been shown to be as prevalent as chronic diseases and to be associated with functional dependency in older adults. ${ }^{6}$ A number of studies have suggested potential associations between geriatric syndromes and certain chronic diseases (e.g., coronary artery disease (CAD), congestive heart failure (CHF), and diabetes mellitus (DM)), ${ }^{6-11}$ but population-based investigations of the co-occurrence of chronic diseases and geriatric syndromes are not available.

To understand how many older adults are potentially managing co-occurring chronic diseases and geriatric syndromes in the United States, a nationally representative data set was analyzed. Three chronic diseases (CAD, CHF, and DM) and two geriatric syndromes (UI, falls) were chosen to function as index conditions to illustrate how frequently older adults may be faced with simultaneous management of diseases and syndromes. Each of these index conditions is highly prevalent in older adults; ${ }^{12,13}$ is associated with 
substantial morbidity, disability, and healthcare utilization; ${ }^{12,14-16}$ is the focus of well-developed disease management guidelines; and has well-defined quality measures to assess its quality of care. ${ }^{17,18}$ The goal of the study was to investigate the co-occurrence of these diseases and syndromes in the older adult population. It was hypothesized that substantial co-occurrence of CAD, CHF, DM, UI, and falls exists in adults aged 65 and older.

\section{METHODS}

\section{Data}

Data were obtained from respondents in the 2004 wave of the Health and Retirement Study (HRS), a longitudinal health interview survey of adults aged 51 and older in the United States. In 1992, the HRS began interviewing adults who were community-dwelling at baseline to obtain detailed self-report information on physical and mental health, financial status, and family support systems. ${ }^{19} \mathrm{Re}-$ spondents are surveyed every 2 years and continue to be followed upon moving into long-term care facilities. Interviews are conducted face to face or over the telephone according to study protocols. If individual respondents are unable to complete the interview, most often because of medical or cognitive conditions, a proxy informant familiar with the respondent's medical conditions and financial status is then interviewed. African-American and Hispanic minority groups are oversampled. For this study, the analyses were limited to respondents aged 65 and older.

The National Institute on Aging sponsors the HRS, which the Institute for Social Research at the University of Michigan conducts. ${ }^{20}$ The Health Sciences Institutional Review Board at the University of Michigan approved the HRS. The data used for this analysis are publicly available and contain no unique identifiers, thus assuring respondent anonymity.

\section{Variables and Their Measurement}

Index Conditions. The 2004 wave of the HRS obtained self-report information on respondents' chronic diseases and geriatric syndromes. Respondents were asked whether a physician had diagnosed them with each disease or whether they had symptoms of each syndrome. For this study, diseases and syndromes were specified as follows:

CAD: physician diagnosis of heart attack or myocardial infarction at any time previously or angina pectoris (or chest pains due to the heart) in the previous 2 years.

CHF: physician diagnosis of CHF in the previous 2 years.

DM: physician diagnosis of DM (or high blood sugar) at any time previously.

UI: loss of urine beyond the respondent's control in the previous year.

Falls: two or more falls or any injurious fall requiring medical attention in the previous 2 years.

\section{Sociodemographic Characteristics}

Sociodemographic variables included respondents' age, sex, race, marital status, living situation (lives alone, with others, or in a long-term care facility), educational attainment, and net worth (total household assets minus current debt).

\section{Statistical Analysis}

The HRS was designed to differentially select for participants so as to be nationally representative. To adjust for its complex sample design, including the differential probability of selection and nonresponse, all analyses were weighted and adjusted using the statistical package STATA (Release 9.2, StataCorp, College Station, TX). Standard descriptive methods were used to estimate the prevalence of and determine confidence intervals for respondents with different numbers of index conditions. Associations between index conditions and demographic characteristics were estimated using the chi-square test.

\section{RESULTS}

Of the 20,129 respondents interviewed in the 2004 wave of the HRS, 11,113 were aged 65 and older, representing 37.1 million older adults nationally. Table 1 shows the sociodemographic characteristics of the study population, weighted to be nationally representative. The respondents were classified according to their number of index conditions $(0,1,2, \geq 3)$. Respondents with more conditions were older; more likely to be female, unmarried, living alone or residing in a nursing facility; and had less education and a lower net worth. No association was found between the number of index conditions and race.

Table 2 shows the prevalence of each index condition and its frequency of co-occurrence with other four conditions. The prevalence of each index condition was: CAD $15.9 \%$, CHF $4.8 \%$, DM $19.4 \%$, UI $25.0 \%$, and falls $23.2 \%$. In general, the chronic diseases and the geriatric syndromes demonstrated substantial co-occurrence. Of the three chronic diseases, CHF had the largest burden of cooccurring conditions.

For respondents not reporting any of the three chronic diseases, the prevalence of the two geriatric syndromes was lower (data not shown). Sixty-four percent did not report either geriatric syndrome, $15 \%$ reported only incontinence, $12 \%$ reported only falls, and $8 \%$ reported incontinence and falls.

Figure 1 illustrates, for each index condition, the prevalence of having no, one, two, or three other conditions. For each index condition, the prevalence of having at least one additional condition exceeded $50 \%$; the prevalence of having two or more additional conditions exceeded $20 \%$. Most striking is the finding that more than $80 \%$ of respondents with CHF had at least one other condition.

\section{DISCUSSION}

This cross-sectional study examined the prevalence of the co-occurrence of three chronic diseases and two geriatric syndromes in the older adult population. These diseases and syndromes are important causes of morbidity in older adults and are targets of management guidelines and clinical quality measures. Previous studies have shown that co-occurring chronic diseases are common in older adults. ${ }^{3}$ The current study, using nationally representative data, furthers these findings by demonstrating that the co-occurrence of chronic diseases with geriatric syndromes is also common. Especially remarkable is the finding that more than $25 \%$ of older adults with any one of the three chronic diseases also had at least one of the two geriatric syndromes. 


\section{Table 1. Respondent Characteristics According to Number of Index Conditions}

Number of Index Conditions, Weighted $\%(N=11,113)$

\begin{tabular}{|c|c|c|c|c|c|}
\hline Characteristic & $0(n=4,861)$ & $1(n=3,715)$ & $2(n=1,777)$ & $\geq 3(n=760)$ & $P$-Value* \\
\hline Overall Prevalence & 43.6 & 33.4 & 16.1 & 6.9 & \\
\hline Age & & & & & $<.001$ \\
\hline$\geq 76(45 \%)$ & 38.1 & 34.0 & 19.6 & 8.4 & \\
\hline Sex & & & & & $<.001$ \\
\hline Male $(42 \%)$ & 46.6 & 33.1 & 14.2 & 6.1 & \\
\hline Race & & & & & .81 \\
\hline White or other $(87 \%)$ & 43.8 & 33.2 & 16.2 & 6.9 & \\
\hline African American (8\%) & 43.1 & 33.6 & 16.2 & 7.1 & \\
\hline Hispanic (5\%) & 42.7 & 35.8 & 14.9 & 6.6 & \\
\hline Married & & & & & $<.001$ \\
\hline $12(36 \%)$ & 45.1 & 33.1 & 14.9 & 6.9 & \\
\hline$>12(36 \%)$ & 46.2 & 33.6 & 15.3 & 4.9 & \\
\hline Net worth, \$ & & & & & $<.001$ \\
\hline $40,000(23 \%)$ & 34.2 & 32.9 & 21.1 & 11.8 & \\
\hline $40,000-154,999(23 \%)$ & 42.6 & 32.8 & 16.7 & 7.9 & \\
\hline $155,000-419,999(26 \%)$ & 47.1 & 33.5 & 13.7 & 5.7 & \\
\hline$\geq 420,000(28 \%)$ & 49.0 & 34.1 & 13.9 & 3.1 & \\
\hline Living status & & & & & $<.001$ \\
\hline Alone (29\%) & 42.9 & 34.5 & 15.6 & 6.9 & \\
\hline With others $(67 \%)$ & 45.6 & 33.3 & 15.2 & 5.9 & \\
\hline
\end{tabular}

Weighted percentages derived using the Health and Retirement Study (HRS) respondent population weights to adjust for the complex sampling design of the HRS survey.

Numbers represent row percentages (i.e., 48.1\% of the respondents aged 65-75 had no index conditions).

* $P$-values are from chi-square test for association between the indicated variable and number of index conditions.

The combination of chronic diseases and geriatric syndromes can have a substantial effect on older adults' functional status and specifically on their ability to manage their overall health. As has been demonstrated, the association between geriatric syndromes and functional disability is as strong as that between chronic diseases and functional disability. ${ }^{6}$ The current study, although employing only five common conditions, highlights that a substantial propor-

Table 2. Prevalence of Individual Co-Occurring Index Conditions

Prevalence of Other Conditions in Respondents with Index Conditions (\%, 95\% Confidence Interval)

Index Condition (Population Prevalence \%)

\section{CAD (15.9)}

CHF (4.8)

DM (19.4)

UI (25.0)

Falls (23.2)

\begin{tabular}{ccccc}
\hline CAD & CHF & DM & UI & Falls \\
\hline- & $17.3(15.4-19.4)$ & $28.6(26.6-30.6)$ & $28.9(26.1-32.1)$ & $34.0(31.3-36.7)$ \\
$57.6(53.7-61.5)$ & - & $36.5(31.3-42.0)$ & $36.7(32.2-41.5)$ & $43.0(38.3-47.7)$ \\
$23.5(21.7-25.4)$ & $9.0(7.6-10.6)$ & - & $28.2(26.1-30.4)$ & $28.8(26.7-30.9)$ \\
$18.5(16.7-20.5)$ & $7.0(6.0-8.3)$ & $21.9(20.1-23.8)$ & - & $36.6(34.6-38.7)$ \\
$23.3(21.3-25.5)$ & $8.9(7.6-10.3)$ & $24.0(22.2-25.9)$ & $39.4(37.2-41.6)$ & - \\
\hline
\end{tabular}

All prevalence percentages were derived using the Health and Retirement Study (HRS) respondent population weights to adjust for the complex sampling design of the HRS survey.

Percentages indicate the prevalence of the other four conditions in respondents with the index condition listed in the first column. For example, in respondents with coronary artery disease (CAD), $17.3 \%$ also have congestive heart failure (CHF), $28.6 \%$ diabetes mellitus (DM), $28.9 \%$ urinary incontinence (UI), and $34.0 \%$ falls. 


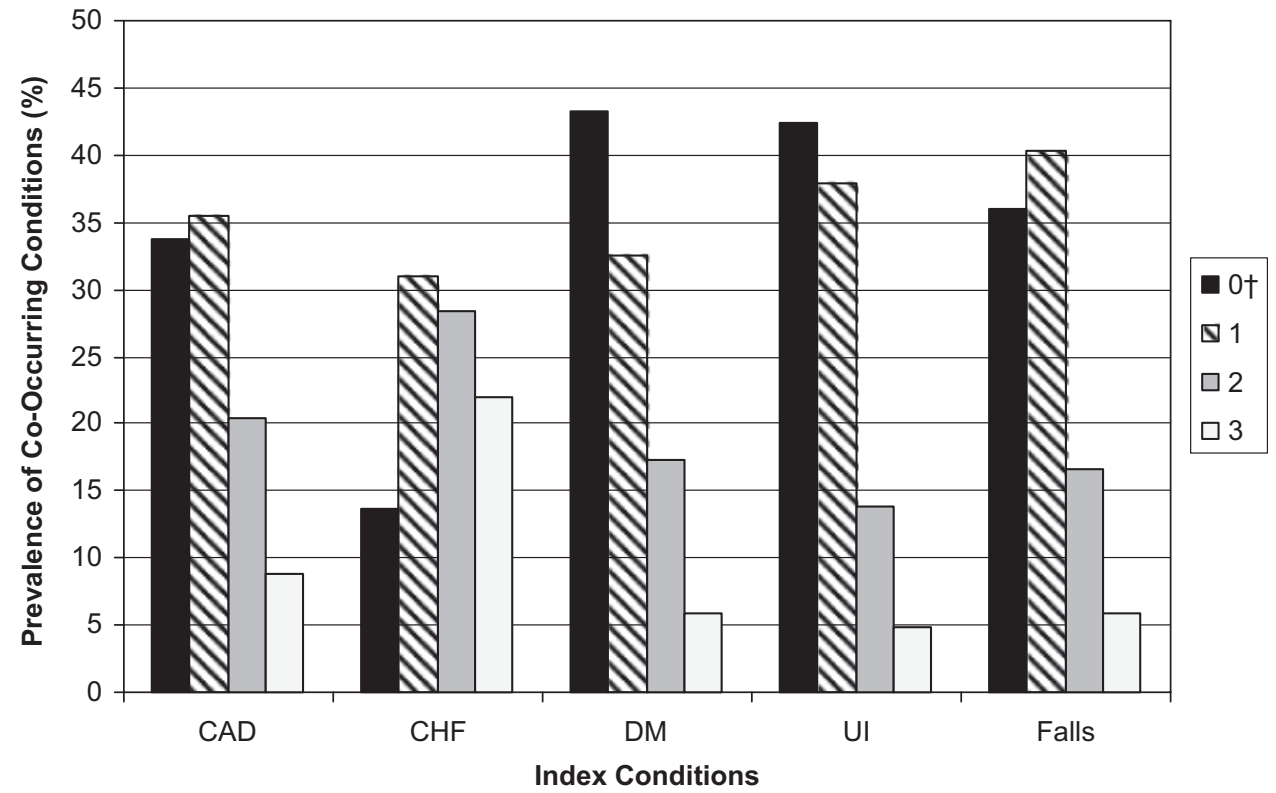

Figure 1. Prevalence of multiple co-occurring index conditions. 'Weighted percentages derived using the Health and Retirement Study (HRS) respondent population weights to adjust for the complex sampling design of the HRS survey. The percentages of respondents with coronary artery disease (CAD), congestive heart failure (CHF), diabetes mellitus (DM), urinary incontinence (UI), or falls who also had no, one, two, or three of the four other index conditions. Y-axis scale is 0 to 50 .

tion of older adults have geriatric syndromes co-occurring with their chronic diseases. Although geriatric syndromes are not routinely considered to be comorbidities, their prevalence, their effect on the functional status of older adults, and their associated risk for poor quality of life suggest that clinically they may be as important as other comorbidities. Thus, physicians should address older adult patients' geriatric syndromes as well as their chronic diseases.

The prevalence rates of the chronic diseases in the study were compared with those reported in the literature. The prevalence of DM in older adults according to HRS data compared favorably with data from the National Health and Nutrition Examination Survey (NHANES, 1999$2004){ }^{21}$ Of adults aged 60 and older in the NHANES, $21 \%$ had DM; $19 \%$ of adults aged 65 years and older had DM in the HRS. In contrast, the prevalence rates for CAD and CHF in the HRS were lower than those for other population-based studies. In the HRS, $16 \%$ of adults aged 65 and older reported $\mathrm{CAD}$, and $5 \%$ reported $\mathrm{CHF}$. In the National Health Interview Survey (2006), 22\% of adults in the same age group reported CAD, and $31 \%$ reported some form of heart disease (CAD and other heart diseases). ${ }^{22}$ For NHANES data, the prevalence of CAD was $32 \%$ for adults aged 80 and older; the prevalence of $\mathrm{CHF}$ was $5 \%$ to $12 \%$ for adults aged 60 and older. ${ }^{21}$ The lower prevalence in the HRS may in part be due to limiting the self-report of angina pectoris and of CHF to the previous 2 years, thereby not including respondents who reported angina pectoris or $\mathrm{CHF}$ before this time period. The aim was to minimize recall bias for these symptoms and diagnoses and thus to make a conservative estimate of the prevalence of CAD and of CHF.

The prevalence rates for the geriatric syndromes in this study were similarly compared with those reported in the literature. The current study found that $25 \%$ of adults aged
65 and older reported having any degree of UI in the previous year, whereas NHANES data yielded a prevalence of $12 \%$ for daily UI for women aged 60 to 64 and $21 \%$ for women aged 85 and older. ${ }^{23}$ The current study found the prevalence of falls to be $23 \%$ of HRS respondents. (Falls were limited to injurious falls and/or two or more falls in the previous 2 years, similar to the criteria for falls in the Physicians Quality Reporting Initiative quality measure used by the Centers for Medicare and Medicaid Services.) Other studies have reported that nearly one-third of older adults fall at least once each year. ${ }^{24,25}$

From a physician and healthcare policy perspective, patients' healthcare quality appears to improve as their number of medical conditions increases, ${ }^{26,27}$ but from a patient perspective, adherence to multiple management guidelines can lead to undue burden for the older adult with multiple diseases and syndromes. It has been found that most health management guidelines do not modify their recommendations or address the applicability of the recommendations for older adults with multiple chronic diseases. $^{3}$

Current indicators of clinical quality are likewise based on individual diseases. Physicians caring for patients with multiple diseases may be motivated to focus on single diseases rather than to provide comprehensive and coordinated care for all of their patients' conditions. Guidelines and clinical performance quality indicators for the geriatric syndromes of UI and falls have been developed and are beginning to be implemented. Although this is an important step, with the potential to improve detection and management of geriatric syndromes, it perpetuates a "one disease or syndrome at a time" management philosophy rather than the integration and coordination of management interventions for patients with complex health status. The current study's findings support the need for the implementation of comprehensive management guidelines and quality indica- 
tors for physicians caring for older adults with multiple diseases and syndromes; such guidelines and indicators of comprehensive clinical quality are now under development. ${ }^{28}$

Finally, from a research standpoint, the co-occurrence of chronic diseases and geriatric syndromes in the current study suggests that they may have shared risk factors. The pathophysiological relationship between DM and heart disease is well known, but the pathways relating chronic diseases to geriatric syndromes have not been fully explored. Future research investigating the causes of the "clustering" of diseases and syndromes would have benefit for prevention, early recognition, and management of accumulating comorbid diseases and syndromes. Such research might lead also to eventual prevention or attenuation of functional disability and decreased quality of life.

A key strength of this study is its use of HRS data. The HRS is a nationally representative survey and includes detailed information on chronic diseases and geriatric syndromes. Although the HRS primarily collects self-report data, it is one of the few nationally representative studies to contain such extensive information on diseases and geriatric syndromes. Self-report data may be less reliable for certain chronic diseases, although research suggests that good agreement exists between validated evidence of myocardial infarction, angina pectoris, $\mathrm{CHF}$, and DM and the selfreport of these diseases. ${ }^{29}$

The current study employs cross-sectional analysis, and causality cannot be determined, although respondents in the HRS are interviewed every 2 years, making possible future longitudinal studies to further evaluate the relationship between chronic diseases and geriatric syndromes. In addition, the prevalence of other common chronic diseases, such as chronic lung disease or arthritis, and other geriatric syndromes, such as delirium, were not analyzed. Rather, diseases and syndromes with well-developed clinical performance indicators, ones on which physicians are now being measured, were selected to highlight the substantial co-occurrence of these five diseases and syndromes. For most primary care physicians and other providers, these five conditions occur commonly in their practices, and these are the most common conditions for which payers and health plans are measuring the quality of clinical care. Future research is needed to more fully explore the effect of multiple other geriatric syndromes on patients with multiple chronic diseases.

\section{CONCLUSION}

It has been convincingly argued that medical practice should evolve to match the changes of an aging population with a longer life expectancy. ${ }^{30}$ Consistent with that view, the results from the current study suggest that, as greater numbers of older adult patients present with multiple cooccurring diseases and geriatric syndromes, physicians will be required to provide comprehensive care to address complex health status. Clinical guidelines and measures of the quality of clinical care must be developed that address coordinated and comprehensive management of multiple co-occurring diseases and syndromes rather than focus on single conditions.

\section{ACKNOWLEDGMENTS}

We would like to thank Ms. Zhiyi Tian for her assistance in statistical analyses.

Conflict of Interest: The editor in chief has reviewed the conflict of interest checklist provided by the authors and has determined that the authors have no financial or any other kind of personal conflicts with this manuscript.

Financial Disclosure: Dr. Lee was supported by the Veterans Affairs (VA) Special Fellowships Program in Advanced Geriatrics through the VA Ann Arbor Healthcare System Geriatric Research, Education and Clinical Center (GRECC); the John A. Hartford Foundation; and the University of Michigan Claude D. Pepper Older Americans Independence Center. Dr. Cigolle was supported by a Ruth L. Kirschstein National Research Service Award from the National Institute on Aging (NIA; 1F32AG027649-01), the National Institutes of Health, National Center for Research Resources K12 Mentored Clinical Scholars Program at the University of Michigan, the VA Ann Arbor Healthcare System GRECC, and the John A. Hartford Foundation. Dr. Blaum was supported by NIA Grant R01 AG021493A and the VA Ann Arbor Healthcare System GRECC. An early version of this paper was presented as a poster at the Gerontological Society of America's Annual Scientific Meeting in November 2007.

Author Contributions: All authors were involved in study concept and design, data analyses, interpretation of data, and preparation of manuscript.

\section{Sponsor's Role: None.}

\section{REFERENCES}

1. Gijsen R, Hoeymans N, Schellevis FG et al. Causes and consequences of comorbidity: A review. J Clin Epidemiol 2001;54:661-674.

2. Wolff JL, Starfield B, Anderson G. Prevalence, expenditures, and complications of multiple chronic conditions in the elderly. Arch Intern Med 2002;162:2269-2276.

3. Boyd CM, Darer J, Boult C et al. Clinical practice guidelines and quality of care for older patients with multiple comorbid diseases: Implications for pay for performance. JAMA 2005;294:716-724.

4. Marengoni A, Winblad B, Karp A et al. Prevalence of chronic diseases and multimorbidity among the elderly population in Sweden. Am J Public Health 2008;98:1198-1200.

5. Wolff JL, Boult C, Boyd C et al. Newly reported chronic conditions and onset of functional dependency. J Am Geriatr Soc 2005;53:851-855.

6. Cigolle CT, Langa KM, Kabeto MU et al. Geriatric conditions and disability: The Health and Retirement Study. Ann Intern Med 2007;147:156-164.

7. Brown JS, Vittinghoff E, Lin F et al. Prevalence and risk factors for urinary incontinence in women with type 2 diabetes and impaired fasting glucose: Findings from the National Health and Nutrition Examination Survey (NHANES) 2001-2002. Diabetes Care 2006;29:1307-1312.

8. Jackson RA, Vittinghoff E, Kanaya AM et al. Urinary incontinence in elderly women: Findings from the Health, Aging, and Body Composition Study. Obstet Gynecol 2004;104:301-307.

9. Rich MW. Heart failure in older adults. Med Clin North Am 2006;90:863885 , xi.

10. Schwartz AV, Hillier TA, Sellmeyer DE et al. Older women with diabetes have a higher risk of falls: A prospective study. Diabetes Care 2002;25:1749-1754.

11. van Gerwen M, Schellevis F, Lagro-Janssen T. Comorbidities associated with urinary incontinence: A case-control study from the Second Dutch National Survey of General Practice. J Am Board Fam Med 2007;20:608-610.

12. Thom T, Haase N, Rosamond W. Heart disease and stroke statistics - 2006 update: A report from the American Heart Association Statistics Committee and Stroke Statistics Subcommittee. Circulation 2006;113:e85-e151.

13. Health, United States, 2005 with Chartbook on Trends in the Health of Americans. Hyattsville, MD: Centers for Disease Control and Prevention, 2005 .

14. Anderson RN, Smith BL Deaths: Leading causes for 2002. Natl Vital Stat Rep 2005;53:1-89. 
15. Elixhauser A, Yu K, Steiner C et al. Hospitalization in the United States, 1997. Agency for Healthcare Research and Quality; AHRQ Publication, Rockville, MD: 2000 .

16. Centers for Disease Control and Prevention, National Center for Injury Prevention and Control [on-line]. Available at http://www.cdc.gov/ncipc/duip/ preventadultfalls.htm Accessed March 18, 2008.

17. Centers for Medicare and Medicaid Services (CMS) [on-line]. Available at http://www.cms.hhs.gov/ Accessed March 18, 2008.

18. NCQA HEDIS and Quality Measurement [on-line]. Available at http:// web.ncqa.org/ Accessed March 18, 2008.

19. The Health and Retirement Study: A Longitudinal Study of Health, Retirement, and Aging; Sponsored by the National Institute of Aging [on-line]. Available at http://hrsonline.isr.umich.edu/ Accessed March 18, 2008.

20. Juster F, Suzman R. The Health and Retirement Study: An Overview. HRS Working Papers Series \#94-1001. J Hum Resources 1995;30:S7-S56.

21. Rosamond W, Flegal K, Furie K et al. Heart disease and stroke statistics-2008 update: A report from the American Heart Association Statistics Committee and Stroke Statistics Subcommittee. Circulation 2008;117:e25e146.

22. Pleis J, Lethbridge-Cejku M. Summary Health Statistics for U.S. Adults: National Health Interview Survey, 2006: U.S. Department of Health and Human
Services, Centers for Disease Control and Prevention, National Center for Health Statistics, 2007. Report No. 235.

23. Anger JT, Saigal CS, Litwin MS. The prevalence of urinary incontinence among community dwelling adult women: Results from the National Health and Nutrition Examination Survey. J Urol 2006;175:601-604.

24. Hornbrook MC, Stevens VJ, Wingfield DJ et al. Preventing falls among community-dwelling older persons: Results from a randomized trial. Gerontologist 1994;34:16-23.

25. Hausdorff JM, Rios DA, Edelberg HK. Gait variability and fall risk in community-living older adults: A 1-year prospective study. Arch Phys Med Rehabil 2001;82:1050-1056

26. Higashi T, Wenger NS, Adams JL et al. Relationship between number of medical conditions and quality of care. N Engl J Med 2007;356:2496-2504.

27. Min LC, Wenger NS, Fung C et al. Multimorbidity is associated with better quality of care among vulnerable elders. Med Care 2007;45:480-488.

28. Brown AF, Mangione CM, Saliba D et al. Guidelines for improving the care of the older person with diabetes mellitus. J Am Geriatr Soc 2003;51:S265-S280.

29. Simpson CF, Boyd CM, Carlson MC et al. Agreement between self-report of disease diagnoses and medical record validation in disabled older women: Factors that modify agreement. J Am Geriatr Soc 2004;52:123-127.

30. Tinetti ME, Fried T. The end of the disease era. Am J Med 2004;116:179-185. 